\title{
ALICE data in the framework of the Color String Percolation Model
}

\author{
Aditya N Mishra*, Eleazar Cuautle, Guy Paić \\ Instituto de Ciencias Nucleares, Universidad Nacional Autónoma de México, \\ Apartado Postal 70-543, México Distrito Federal 04510, México \\ E-mail:Aditya.Nath.Mishra @ cern.ch \\ E-mail:Eleazar.Cuautle.Flores@cern.ch E-mail:Guy.Paic@cern.ch
}

\section{Carlos Pajares}

Departamento de Fisica de Particulas, Universidale de Santiago de Compostela and Instituto Galego de Fisica de Atlas Enerxias(IGFAE), 15782 Santiago, de Compostela, Spain

E-mail:pajares@fpaxp1.usc.es

\section{Rolf P Scharenberg, Brijesh K Srivastava}

Department of Physics and Astronomy, Purdue University, West Lafayette, IN-47907, USA

E-mail: schrnbrg@purdue.edu

E-mail: brijesh@purdue.edu

Possible phase transition of strongly interacting matter from hadron to a quark-gluon plasma state have in the past received considerable interest. The clustering of color sources provides a framework of the partonic interactions in the initial stage of the collisions. The onset of deconf nement transition is identif ed by the spanning percolation cluster in $2 \mathrm{D}$ percolation. In the present work we have analyzed the transverse momentum spectra of charged particles in high multiplicity pp collisions at LHC energies $\overline{\mathrm{s}}=5.02$ and $13 \mathrm{TeV}$ published by the ALICE Collaboration using the Color String Percolation Model (CSPM). For heavy ions PbPb at $\overline{\mathrm{S}_{\mathrm{NN}}}$ $=2.76$ and $5.02 \mathrm{TeV}$ along with XeXe at $\overline{\mathrm{S}_{\mathrm{NN}}}=5.44 \mathrm{TeV}$ have been analyzed.

The thermodynamical quantities, the temperature, energy density, and the shear viscosity to entropy density ratio $(\eta / s)$ are obtained. It was observed that the inverse of $(\eta / s)$ represents the trace anomaly $\Delta=(\varepsilon-3 \mathrm{P}) / \mathrm{T}^{4}$. Results are in agreement with the Lattice Quantum Chromo Dynamics(LQCD) simulations. Thus the clustering of color sources has a clear physical basis although it cannot be deduced directly from QCD.

7th Edition of the Large Hadron Collider Physics Conference,

20-25 May 2019

Puebla, Mexico

\footnotetext{
${ }^{*}$ Speaker.
} 


\section{Introduction}

The observation of high total multiplicity, high transverse energy, non-jet and isotropic events led Van Hove to conclude that high energy density events are produced in high energy $\bar{p} p$ collisions [1]. In these events the transverse energy is proportional to the number of low transverse momentum particles. This basic correspondence has been previously explored over a wide range of the charged particle pseudorapidity density $\left\langle d N_{c} / d \eta\right\rangle$ in $\bar{p} p$ collisions at center of mass energy $\sqrt{s}=$ $1.8 \mathrm{TeV}$ [2]. The multiplicity independent freezout energy density $\sim 1.1 \mathrm{GeV} / \mathrm{fm}^{3}$ at a temperature of $\sim 179 \mathrm{MeV}$ further suggested deconfinement in $\bar{p} p$ collisions [3].

The objective of the present work is to obtain the initial temperature and the shear viscosity to entropy density ratio $\eta / s$ of the matter created in $p p$ collisions at LHC energies by analyzing the published ALICE data on the transverse momentum spectra of charged hadrons in $p p, \mathrm{XeXe}$, and $\mathrm{PbPb}$ collisions using the framework of clustering of color sources $[4,5,6]$.
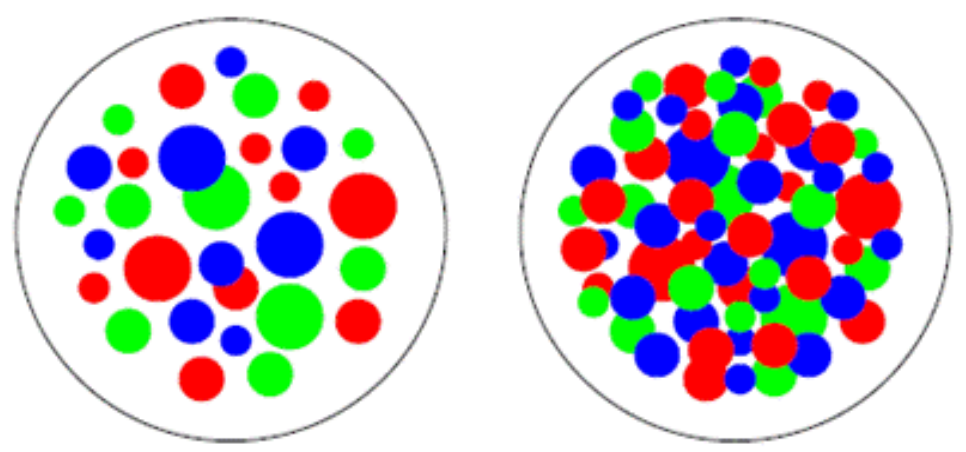

Figure 1: Partonic cluster structure in the transverse collision plane at low (left) and (right) high parton density [7].

All high energy soft multi-hadron interactions exhibit thermal patterns of abundances characterized by the same temperature, independent of the center of mass energy $[8,9]$. The hadron limiting temperatures were measured by statistical thermal analyses that fit the data with a minimum of parameters $[8,9]$.

\section{Clustering of Color Sources}

Multi-particle production at high energies is currently described in terms of color strings stretched between the projectile and target. Hadronizing these strings produce the observed hadrons. At low energies only valence quarks of nucleons form strings that then hadronize. The number of strings grows with the energy and with the number of nucleons of participating nuclei. Color strings may be viewed as small discs in the transverse space filled with the color field created by colliding partons, very much similar to disks in two dimensional percolation theory as shown in Fig. $1[7,10]$. Particles are produced by the Schwinger mechanisms [11]. With growing energy and size of the colliding nuclei the number of strings grows and starts to overlap to form clusters $[12,13,14]$. At a critical density a macroscopic cluster appears that marks the percolation phase transition. This is termed as Color String percolation Model (CSPM) [12, 13]. The interaction between strings occurs when they overlap and the general result, due to the $\mathrm{SU}(3)$ random summation 
of charges, is a reduction in the multiplicity and an increase in the string tension or an increase in the average transverse momentum squared, $\left\langle p_{t}^{2}\right\rangle$. We assume that a cluster of $n$ strings that occupies an area of $S_{n}$ behaves as a single color source with a higher color field $\vec{Q}_{n}$ corresponding to the vectorial sum of the color charges of each individual string $\vec{Q}_{1}$. The resulting color field covers the area of the cluster. As $\vec{Q}_{n}=\sum_{1}^{n} \vec{Q}_{1}$, and the individual string colors may be oriented in an arbitrary manner respective to each other, the average $\overrightarrow{Q_{1 i}} \overrightarrow{Q_{1 j}}$ is zero, and $\vec{Q}_{n}^{2}=n \overrightarrow{Q_{1}^{2}}$.

Knowing the color charge $\vec{Q}_{n}$ one can obtain the multiplicity $\mu$ and the mean transverse momentum squared $\left\langle p_{t}^{2}\right\rangle$ of the particles produced by a cluster of $n$ strings [13]

$$
\mu_{n}=\sqrt{\frac{n S_{n}}{S_{1}}} \mu_{0} ; \quad\left\langle p_{t}^{2}\right\rangle=\sqrt{\frac{n S_{1}}{S_{n}}}\left\langle p_{t}^{2}\right\rangle_{1}
$$

where $\mu_{0}$ and $\left\langle p_{t}^{2}\right\rangle_{1}$ are the mean multiplicity and $\left\langle p_{t}^{2}\right\rangle$ of particles produced from a single string with a transverse area $S_{1}=\pi r_{0}^{2}$. In the thermodynamic limit, one obtains an analytic expression $[12,13]$

$$
\left\langle\frac{n S_{1}}{S_{n}}\right\rangle=\frac{\xi}{1-e^{-\xi}} \equiv \frac{1}{F(\xi)^{2}} ; \quad F(\xi)=\sqrt{\frac{1-e^{-\xi}}{\xi}}
$$

where $F(\xi)$ is the color suppression factor. $\xi=\frac{N_{S} S_{1}}{S_{N}}$ is the percolation density parameter assumed to be finite when both the number of strings $N_{S}$ and total interaction area $S_{N}$ are large. Eq. (2.1) can be written as $\mu_{n}=F(\xi) \mu_{0}$ and $\left\langle p_{t}^{2}\right\rangle_{n}=\left\langle p_{t}^{2}\right\rangle_{1} / F(\xi)$. The critical cluster which spans $S_{N}$, appears for $\xi_{c} \geq 1.2$ [15].

It is worth noting that CSPM is a saturation model similar to the Color Glass Condensate (CGC), where $\left\langle p_{t}^{2}\right\rangle_{1} / F(\xi)$ plays the same role as the saturation momentum scale $Q_{s}^{2}$ in the CGC $[16,17]$.

\section{Color Suppression Factor $F(\xi)$}

In our earlier work $F(\xi)$ was obtained in AuAu collisions by comparing the charged hadron transverse momentum spectra from $p p$ and AuAu collisions[14]. To evaluate the initial value of $F(\xi)$ from data for AuAu collisions, a parameterization of the experimental data of $p_{t}$ distribution in $p p$ collisions $\sqrt{s}=200 \mathrm{GeV}$ was used [14]. The charged particle spectrum is described by a power law [14]

$$
d^{2} N_{c} / d p_{t}^{2}=a /\left(p_{0}+p_{t}\right)^{\alpha},
$$

where $a$ is the normalization factor, $p_{0}$ and $\alpha$ are fitting parameters with $p_{0}=1.98$ and $\alpha=12.87$ [14]. This parameterization is used in high multiplicity $p p$ collisions to take into account the interactions of the strings [14]

$$
\frac{d^{2} N_{c}}{d p_{T}^{2}}=\frac{a}{\left(p_{0} \sqrt{F(\xi)_{p p} / F(\xi)_{p p}^{m u l t}}+p_{T}\right)^{\alpha}} .
$$

where $F(\xi)_{p p}^{m u l t}$ is the multiplicity dependent color suppression factor. In $p p$ collisions $F(\xi)_{p p} \sim$ 1 at low energies due to the low overlap probability. 
The spectra were fitted using Eq. (3.2) in the softer sector with $p_{t}$ in the range $0.12-1.0 \mathrm{GeV} / \mathrm{c}$. Figure 2 shows the Color Suppression Factor $F(\xi)$ in $p p, P b P b$ and $X e X e$ collisions vs $d N_{c h} / d \eta$ scaled by the transverse area $S_{\perp}$. For $p p$ collisions $S_{\perp}$ is multiplicity dependent as obtained from IP-Glasma model [18]. In case of $\mathrm{PbPb}$ and $\mathrm{XeXe}$ collisions the nuclear overlap area was obtained using the Glauber model [19].

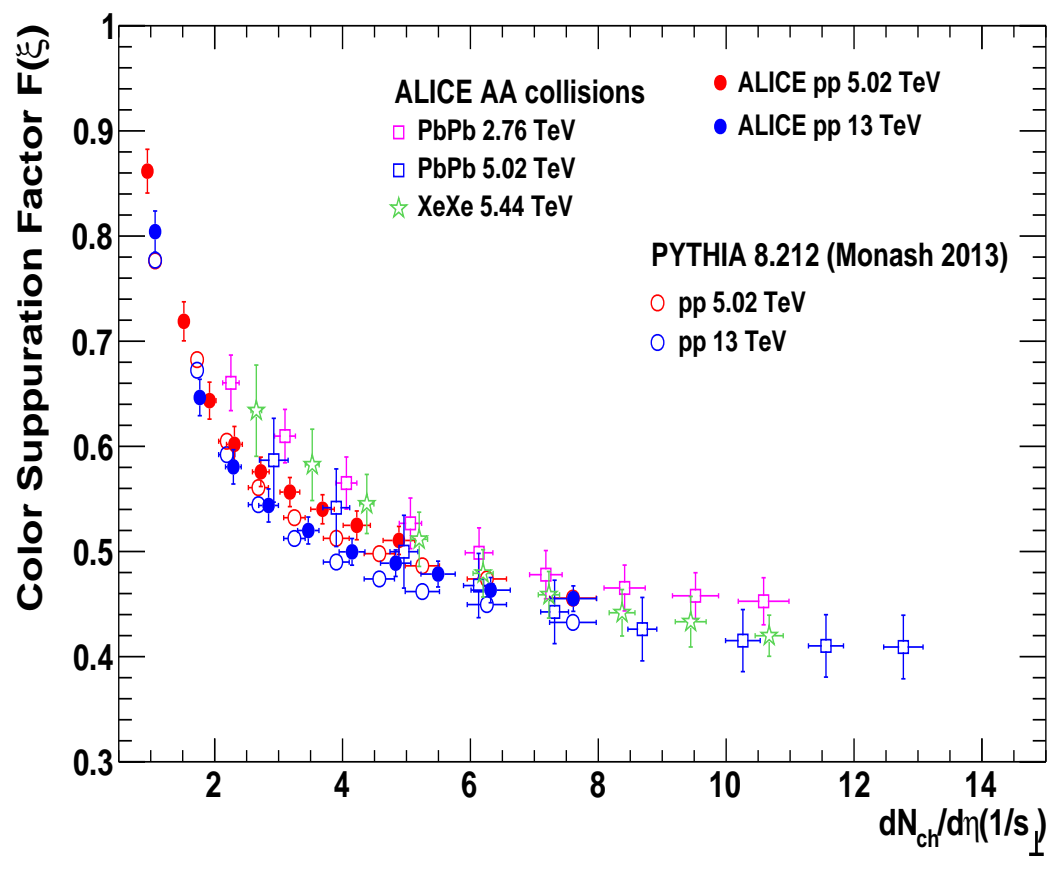

Figure 2: Color Suppression Factor $F(\xi)$ in $p p, P b P b$ and $X e X e$ collisions vs $d N_{c h} / d \eta$ scaled by the transverse area $S_{\perp}$. For $p p$ collisions $S_{\perp}$ is multiplicity dependent as obtained from IP-Glasma model [18]. In case of $\mathrm{PbPb}$ and $\mathrm{XeXe}$ collisions the nuclear overlap area was obtained using the Glauber model [19].

\section{Temperature measurement and thermalization}

The connection between $F(\xi)$ and the temperature $T(\xi)$ involves the Schwinger mechanism (SM) for particle production. The Schwinger distribution for massless particles is expressed in terms of $p_{t}^{2}$ [20]

$$
d n / d p_{t}^{2} \sim \exp \left(-\pi p_{t}^{2} / x^{2}\right)
$$

where the average value of the string tension is $\left\langle x^{2}\right\rangle$. The tension of the macroscopic cluster fluctuates around its mean value because the chromo-electric field is not constant. The origin of the string fluctuation is related to the stochastic picture of the QCD vacuum. Since the average value of the color field strength must vanish, it cannot be constant but changes randomly from point to point [21]. Such fluctuations lead to a Gaussian distribution of the string tension

$$
\frac{d n}{d p_{t}^{2}} \sim \sqrt{\frac{2}{<x^{2}>}} \int_{0}^{\infty} d x \exp \left(-\frac{x^{2}}{2<x^{2}>}\right) \exp \left(-\pi \frac{p_{t}^{2}}{x^{2}}\right)
$$


which gives rise to thermal distribution [21]

$$
\frac{d n}{d p_{t}^{2}} \sim \exp \left(-p_{t} \sqrt{\frac{2 \pi}{\left\langle x^{2}\right\rangle}}\right),
$$

with $\left\langle x^{2}\right\rangle=\pi\left\langle p_{t}^{2}\right\rangle_{1} / F(\xi)$. The temperature is expressed as [14]

$$
T(\xi)=\sqrt{\frac{\left\langle p_{t}^{2}\right\rangle_{1}}{2 F(\xi)}} .
$$

The string percolation density parameter $\xi$ which characterizes the percolation clusters measures

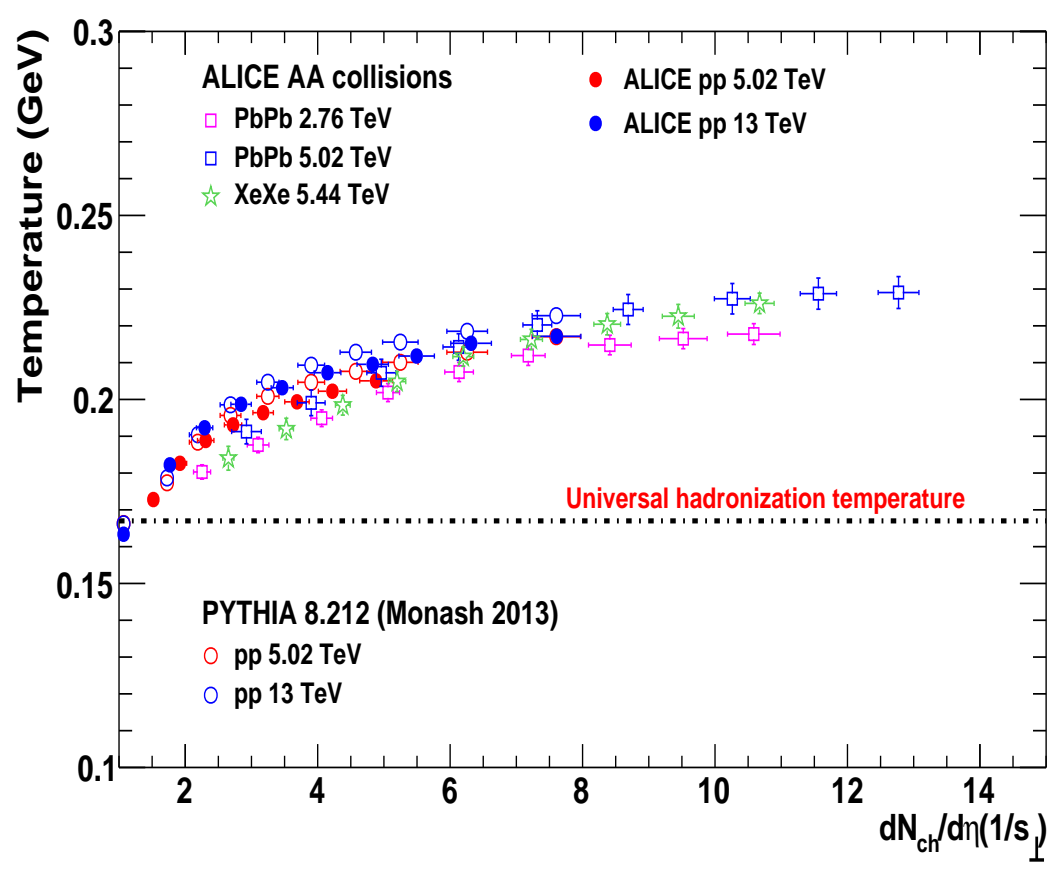

Figure 3: Temperature vs $d N_{c h} / d \eta$ scaled by $S_{\perp}$ from $p p, P b P b$ and $X e X e$ collisions. The horizontal line at $\sim 165 \mathrm{MeV}$ is the universal hadronization temperature [9].

the initial temperature of the system. Since this cluster covers most of the interaction area, the temperature becomes a global temperature determined by the string density. In this way at $\xi_{c}=1.2$ the connectivity percolation transition at $T\left(\xi_{c}\right)$ models the thermal deconfinement transition.

Figure 3 shows a plot of temperature as a function of $N_{\text {tracks }} / \Delta \eta$ scaled by $S_{\perp}$. The horizontal line at $\sim 165 \mathrm{MeV}$ is the universal hadronization temperature obtained from the systematic comparison of the statistical model parametrization of hadron abundances measured in high energy $e^{+} e^{-}, p p$, and $A A$ collisions [9]. The temperatures obtained in higher multiplicity events are consistent with the creation of deconfined matter in $p p$ collisions at $\sqrt{s}=5.02$ and $13 \mathrm{TeV}$. The thermalization in $p p$ collisions can occur through the existence of an event horizon caused by a rapid deceleration of the colliding nuclei. The thermalization in this case is due the HawkingUnruh effect $[22,23,24,25]$. In CSPM the strong color field inside the large cluster produces 


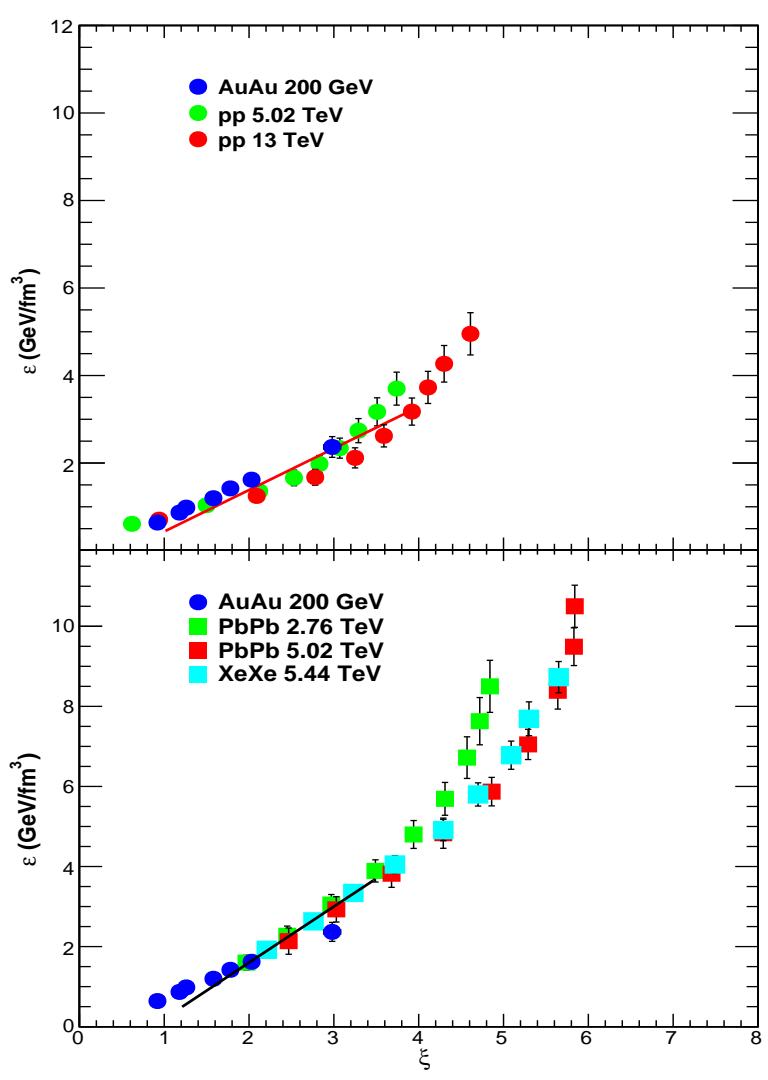

Figure 4: Energy density $(\varepsilon)$ as a function of the percolation density parameter $(\xi)$. Upper panel shows the pp collision data at $\sqrt{s}=5.02$ and $13 \mathrm{TeV}$. Lower panel shows the data for $\mathrm{PbPb}$ at $\sqrt{s_{N N}}=2.76$ and 5.02 $\mathrm{TeV}$ and XeXe data at $\sqrt{s_{N N}}=5.44 \mathrm{TeV}$.

de-acceleration of the primary $q \bar{q}$ pair which can be seen as a thermal temperature by means of Hawking-Unruh effect. This implies that the radiation temperature is determined by the transverse extension of the color flux tube/cluster in terms of the string tension [25].

$$
T=\sqrt{\frac{\sigma}{2 \pi}}
$$

\section{Energy Density}

Among the most important and fundamental problems in finite-temperature QCD are the calculation of the bulk properties of hot QCD matter and characterization of the nature of the QCD phase transition. The QGP according to CSPM is born in local thermal equilibrium because the temperature is determined at the string level. After the initial temperature $T>T_{c}$ the CSPM perfect fluid may expand according to Bjorken boost invariant 1D hydrodynamics [26]

$$
\varepsilon=\frac{3}{2} \frac{\frac{d N_{c}}{d y}\left\langle m_{t}\right\rangle}{S_{n} \tau_{p r o}}
$$


where $\varepsilon$ is the energy density, $S_{n}$ nuclear overlap area, and $\tau$ the proper time.

$$
\tau_{\text {pro }}=\frac{2.405 \hbar}{\left\langle m_{t}\right\rangle}
$$

Above the critical temperature only massless particles are present in CSPM. From the measured value of $\xi$ and $\varepsilon$, as shown in Fig. 4, it is found that $\varepsilon$ is proportional to $\xi$ for the range $1.2<\xi<$ 4.0. Above $\xi \sim 4$ the energy density $\varepsilon$ rises faster compared to $\xi<4$.

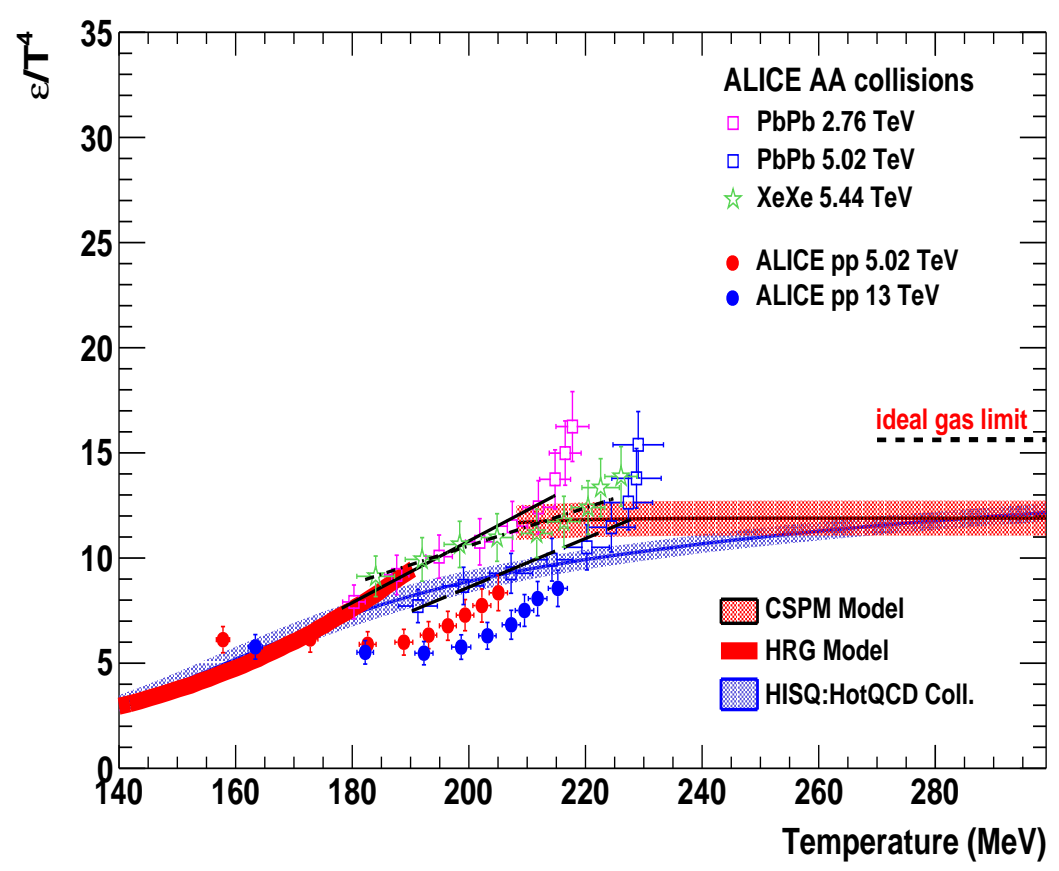

Figure 5: Dimensionless quantity $\varepsilon / T^{4}$ as a function of temperature from CSPM and LQCD calculation from HotQCD Collaboration [27]. The CSPM values at higher temperature T $>200 \mathrm{MeV}$ are obtained extrapolating from lower temperature.

Energy density has been obtained in lattice set up of (2+1)-flavor QCD using the HISQ action and the tree-level improved gauge action [27]. Figure 5 shows dimensionless quantity $\varepsilon / T^{4}$ as a function of temperature both from CSPM and LQCD.

\section{Shear Viscosity to entropy density ratio $\eta / s$ and Trace anomaly $\Delta$}

The relativistic kinetic theory relation for the shear viscosity over entropy density ratio, $\eta / s$ is given by [28]

$$
\frac{\eta}{S} \simeq \frac{T \lambda_{m f p}}{5}
$$

where $\mathrm{T}$ is the temperature and $\lambda_{m f p}$ is the mean free path. $\lambda_{m f p} \sim \frac{1}{\left(n \sigma_{t r}\right)}$ where $n$ is the number density of an ideal gas of quarks and gluons and $\sigma_{t r}$ the transport cross section. In CSPM the 
number density is given by the effective number of sources per unit volume [29]

$$
n=\frac{N_{\text {sources }}}{S_{N} L}
$$

$\mathrm{L}$ is the longitudinal extension of the source, $\mathrm{L}=1 \mathrm{fm} . \eta / \mathrm{s}$ is obtained from $\xi$ and the temperature

$$
\frac{\eta}{s}=\frac{T L}{5\left(1-e^{-\xi}\right)}
$$

Figure 6(upper plot) shows $\eta / s$ as a function of the temperature. The lower bound shown in

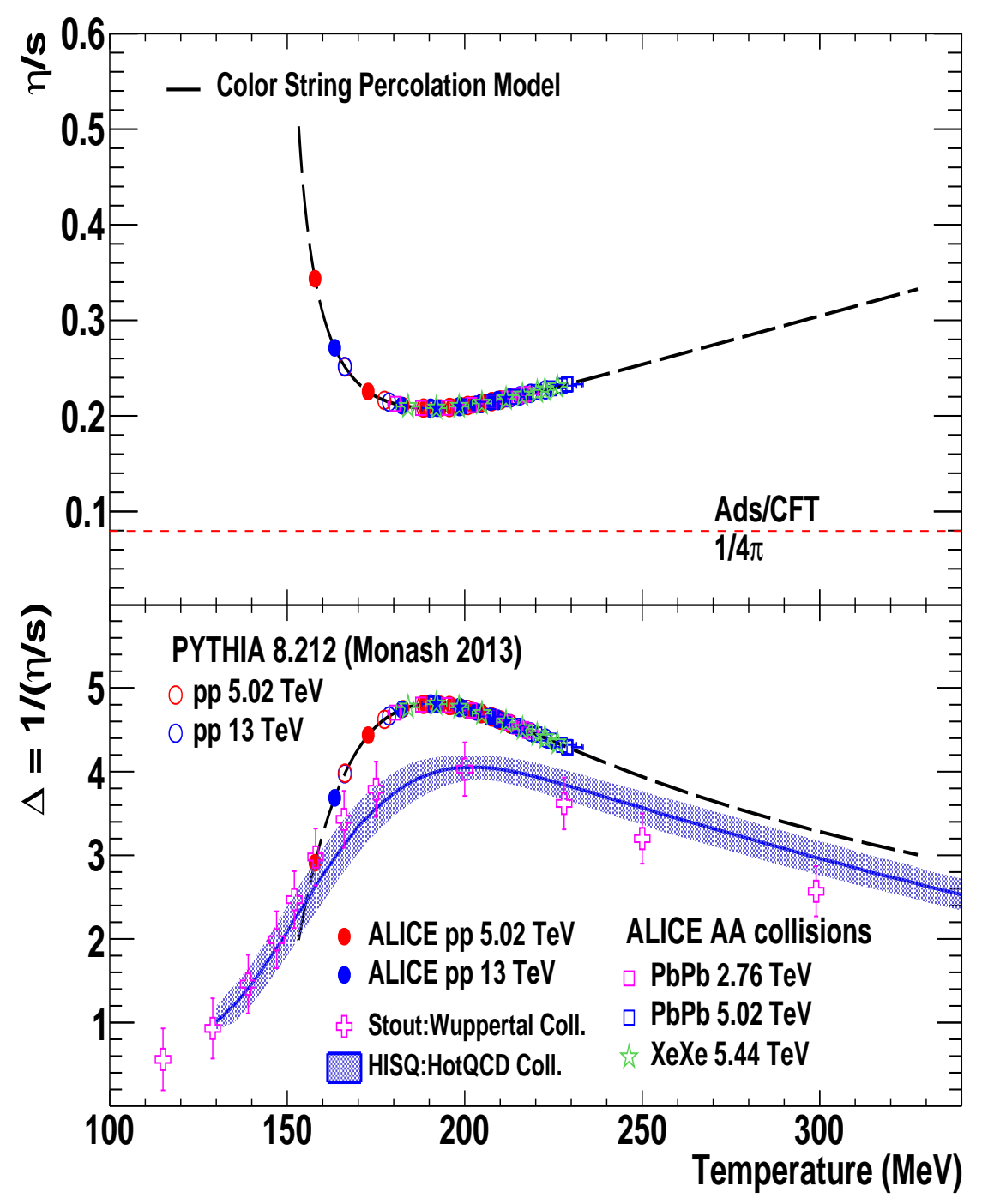

Figure 6: (Upper plot). $\eta / s$ as a function of temperature T using Eq. (6.3) for $\sqrt{s}=5.02$ and $13 \mathrm{TeV}$. The lower bound shown is given by the AdS/CFT [30]. For comparison purposes the results from $\mathrm{PbPb}$ and $\mathrm{XeXe}$ are also shown in the figure.

(Lower plot). The trace anomaly $\Delta=(\varepsilon-3 p) / T^{4}$ vs temperature. Blue open squares are from HotQCD Collaboration [27]. Black stars are from Wuppertal Collaboration [31]. The CSPM results are obtained as $\Delta=1 /(\eta / s)[14]$. The black dashed line corresponds to extrapolation from CSPM at higher temperatures. 
Fig. 6(upper plot) is given by the AdS/CFT conjecture [30]. The results from $p p$ collisions from $\sqrt{s}=13 \mathrm{TeV}$ shows a very small $\eta / s$ and that is 2.7 times the AdS/CFT conjectured lower bound $1 / 4 \pi$.

The trace anomaly $(\Delta)$ is the expectation value of the trace of the energy-momentum tensor, $\left\langle\Theta_{\mu}^{\mu}\right\rangle=(\varepsilon-3 p)$, which measures the deviation from conformal behavior and thus identifies the interaction still present in the medium [27]. We consider the ansatz that inverse of $\eta / s$ is equal to the trace anomaly $\Delta$. This result is shown in Fig. 6(lower plot). The inverse of $\eta / s$ is in quantitative agreement with $(\varepsilon-3 p) / T^{4}$ over a wide range of temperatures. The minimum in $\eta / s \sim 0.20$ determines the peak of the interaction measure $\sim 5$ in agreement with the recent HotQCD values [27]. This happens at the critical temperature of $T_{c} \sim 175 \mathrm{MeV}$. The results from Wuppertal Collaboration is also shown in Fig. 6(lower plot) [31].

\section{Equation of State EOS : The sound velocity $C_{s}^{2}$}

An analytic expression for the equation of state, the sound velocity $C_{s}^{2}$ is obtained in CSPM. After the initial temperature $T>T_{c}$ the CSPM perfect fluid may expand according to Bjorken boost invariant $1 \mathrm{D}$ hydrodynamics [26]. The input parameters are the initial temperature $\mathrm{T}$, the initial energy density $\varepsilon$, and the trace anomaly $\Delta$ are determined by data. The Bjorken 1D expansion gives the sound velocity

$$
\begin{aligned}
\frac{1}{T} \frac{d T}{d \tau} & =-C_{s}^{2} / \tau \\
\frac{d \varepsilon}{d \tau} & =-T s / \tau
\end{aligned}
$$

where $\varepsilon$ is the energy density, s the entropy density, $\tau$ the proper time, and $C_{s}$ the sound velocity. One can eliminate $\tau$ using above expressions to obtain sound velocity as

$$
\begin{array}{r}
s=\left(1+C_{s}^{2}\right) \frac{\varepsilon}{T} \\
\frac{d T}{d \varepsilon} s=C_{s}^{2} .
\end{array}
$$

Since $s=(\varepsilon+P) / T$ and $P=\left(\varepsilon-\Delta T^{4}\right) / 3$ one can express $C_{s}^{2}$ in terms of $\xi$

$$
C_{s}^{2}=\left(\frac{\xi e^{-\xi}}{1-e^{-\xi}}-1\right)\left(-\frac{1}{3}+\frac{\Delta}{12} \times \frac{1}{N}\right),
$$

In Eq. (7.5) $\mathrm{N}=\varepsilon / T^{4}$ and is obtained from Fig.5. The sound velocity squared $C_{s}^{2}$ as obtained using Eq. (7.5) is shown in Fig. 7 as a function of temperature along with LQCD simulations [27].

\section{Conclusion}

We have used the Color String Percolation Model (CSPM) to compute the thermodynamics of the initial stage of $p p$ collisions at LHC energies for temperature, for the shear viscosity to entropy density ratio, the trace anomaly and the sound velocity. The data are obtained from published 


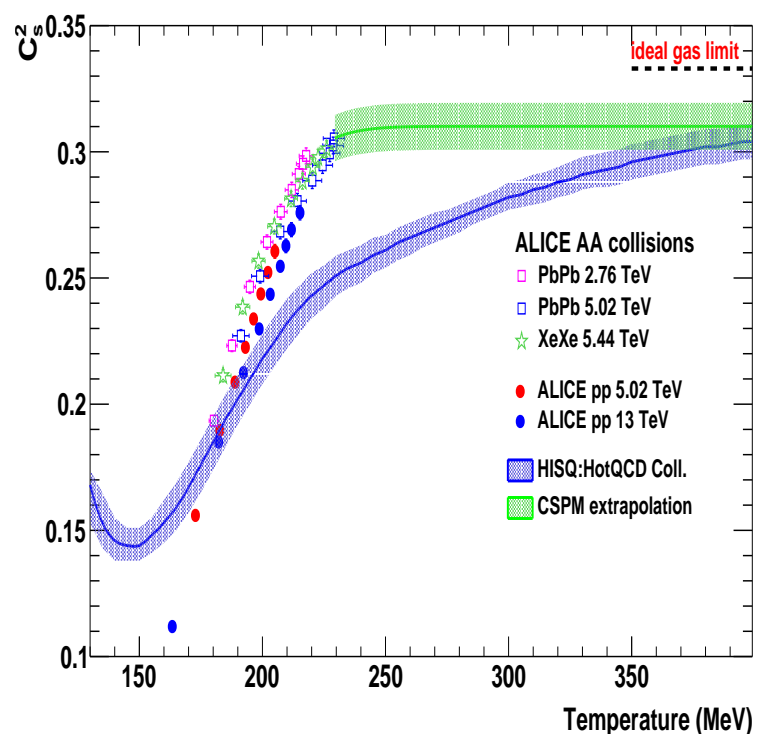

Figure 7: The speed of sound from CSPM and Lattice QCD-p4 versus Temperature [27].

ALICE results at $\sqrt{s}=5.02$ and $13 \mathrm{TeV}$. A universal scaling of the color reduction factor is obtained for both $p p$ and $A A$. For high multiplicity events in $p p$ collisions the temperature is well above the universal hadronization temperature indicating that the matter created is in the deconfined phase. The thermalization in both $p p$ and $A u A u$ is reached through the stochastic process ( HawkingUnruh) rather than kinetic approach. The small $\eta / s$ near the transition temperature also suggests the formation of a strongly coupled $Q G P$.

The main assumption of the present approach is that the inverse of $\eta / s$ represents the trace anomaly, $\Delta=(\varepsilon-3 p) / T^{4}$. The clustering of color sources (percolation) provides us with a microscopic partonic picture that connects the transport properties of the QGP to its thermodynamics.

\section{Acknowledgment}

A.M. acknowledges the post-doctoral fellowship of DGAPA UNAM. Partial support was received by DGAPA-PAPIIT IN109817 and CONACYT A1-S-16215 projects. C.P. thanks the grant Maria de Maeztu Unit of excelence MDM-2016-0682 of Spain, the support of Xunta de Galicia under the project ED431C 2017 and project FPA 2017-83814 of Ministerio de Ciencia e Innovacion of Spain and FEDER.

\section{References}

[1] L. V. Hove, Phys. Lett. B 118, 138 (1982).

[2] T. Alexopoulos et al. (E735 Collaboration), Phys. Rev. D 48, 984 (1993).

[3] T. Alexopoulos et al., Phys. Lett. B 528, 43 (2002).

[4] S. Acharya et al. (ALICE Collaboration), arXiv:1905.07208.

[5] J. Acharya et al. (ALICE Collaboration), JHEP 11, 013 (2018).

[6] J. Adam et al. (ALICE Collaboration), Phys. Lett. B 788, 166 (1019). 
[7] H. Satz, Extreme States of Matter in Strong Interaction Physics, Lecture Notes in Physics 841 ( Springer 2012).

[8] P. Braun-Munzinger, J. Stachel, Christof Wetterich, Phys. Lett. B596, 61 (2004).

[9] F. Becattini, P. Castorina, A. Milov, H. Satz, Eur. Phys. J. C66, 377 (2010).

[10] M. B. Isichenko, Rev. Mod. Phys.64, 961 (1992).

[11] J. Schwinger, Phys. Rev. 82, 664 (1951).

[12] M. A. Braun, C. Pajares, Eu. Phys. J. C16, 349 (2000).

[13] M. A. Braun, F. del Moral, C. Pajares, Phys. Rev. C 65, 024907 (2002).

[14] M. A. Braun et al., Phys. Rep. 599, 1 (2015).

[15] H. Satz, Rep. Prog. Phys. 63, 1511 (2000).

[16] L. McLerran, R. Venugopalan, Phys. Rev. D49, 2233 (1994); 3352 (1994).

[17] J. Dias de Deus and C. Pajares, Phys. Lett. B695, 455 (2011).

[18] L. McLerran, M. Praszalowicz and B. Schenke, Nucl. Phys. A 916, 210 (2013).

[19] C. Loizides, Phys. Rev. C 94, 024914 (2016).

[20] C. Y. Wong, Introduction to high energy heavy ion collisions (World Scientific,1994).

[21] A. Bialas, Phys. Lett. B466, 301 (1999).

[22] D. Kharzeev and E. Levin and K. Tuchin, Phys. Rev. C75, 044903 (2007).

[23] S. W. Hawking, Commun. Math. Phys. 43,199 (1975).

[24] W. G. Unruh, Phys. Rev. D14, 870 (1976).

[25] P. Castorina, D. Kharzeev and H. Satz, Eur. Phys. J. C 52, 187 (2007).

[26] J. D. Bjorken, Phys. Rev. D27, 140 (1983).

[27] A. Bazavov et al.,Phys. Rev. D 90, 094503 (2014).

[28] T. Hirano and M. Gyulassy, Nucl. Phys. A 769, 71 (2006).

[29] J. Dias de Deus, A. S. Hirsch, C. Pajares, R. P. Scharenberg, B. K. Srivastava, Eur. Phys. J. C72, 2123 (2012).

[30] P. K. Kovtun, D. T. Son, A. O. Starinets, Phys. Rev. Lett. C 94, 111601 (2005).

[31] S. Borsanyi et al., Phys. Lett. 730, 99 (2014). 